\title{
A rare case report of psoas abscess in pregnancy in a tertiary centre
}

\section{Niranjan N. Chavan, Umme Ammara*, Zaneta Dias, Manan Boob}

Department of Obstetrics and Gynecology, LTMMC, Sion, Mumbai, Maharashtra, India

Received: 15 November 2021

Revised: 07 December 2021

Accepted: 08 December 2021

\author{
*Correspondence: \\ Dr. Umme Ammara, \\ E-mail: ummeammara94@gmail.com
}

Copyright: () the author(s), publisher and licensee Medip Academy. This is an open-access article distributed under the terms of the Creative Commons Attribution Non-Commercial License, which permits unrestricted non-commercial use, distribution, and reproduction in any medium, provided the original work is properly cited.

\section{ABSTRACT}

A psoas abscess in pregnancy is a relatively uncommon condition with nonspecific signs and symptoms. It may lead to serious complications if not diagnosed and treated promptly. Although spinal tuberculosis affects nearly half of skeletal tuberculosis patients, psoas abscess develops in only $5 \%$ of spinal tuberculosis cases. A clinical history and examination are used to make a diagnosis, which is then confirmed by microbiology and radiological findings. Here is an interesting case report on psoas abscess in pregnancy managed by pigtail catheter insertion and drainage.

Keywords: Psoas abscess, Pregnancy, Spinal tuberculosis

\section{INTRODUCTION}

Psoas abscess is rare during pregnancy. ${ }^{1}$ Although spinal tuberculosis affects nearly half of skeletal tuberculosis patients, psoas abscess develops in only $5 \%$ of spinal tuberculosis cases. ${ }^{2}$ Two types of psoas abscesses exist, the primary and secondary abscess. Primary abscess is caused by spread of pathogen by haematogenous route. On the other hand secondary abscess result from a direct extension of an infectious process into psoas muscle. Most common sites of initial focus include digestive tract (Crohn's abscess, appendicitis and diverticulitis), osteomyelitis, pyelitis, tuberculosis. Other secondary pathogenic organisms responsible for this condition include $S$. aureus, E. coli, bacteroides, and enterococcus amongst others. ${ }^{3}$

We present a case of a primary psoas muscle abscess in pregnancy diagnosed incidentally for which pigtail catheter drainage was done.

\section{CASE REPORT}

A 22 year old primigravida with 7.1 weeks gestation came with complaint of increase in size of abdomen, heaviness in abdomen since 2 months. She had complaints of constipation and tenesmus since 2 months, which was not associated with any pain in abdomen. Patient noticed a lump in abdomen 1 week back after her ultrasound was done. Urine pregnancy test (UPT) was positive. Patient had an ultrasound report suggestive of complex ovarian mass for which tumour markers were done which came out to be normal. As the origin of mass was not confirmed, MRI was done which was suggestive of right psoas abscess of $24.7 \times 12.4 \times 8 \mathrm{~cm}$ extending from $\mathrm{T} 12$ to pelvis extending into the proximal right thigh along the iliopsoas tendon. It was focally eroding the anterior and right lateral surface of T12 and L1 vertebra. Multiple enlarged necrotic lymph nodes were noted in portal hilar, left para-aortic and peri-pancreatic regions.

Patient did not have any pain while movement or on walking.

Patient had a past history of cervical and spinal tuberculosis 10 years back for which some procedure was done on her back in thoracic region, papers not available (scar seen). 6 months of isoniazid, rifampicin, ethambutol and pyrazinamide (AKT) taken. There was no other high risk. 
On physical examination, patient was afebrile. Examination of respiratory and cardiovascular systems was normal. Abdominal examination revealed a 24 weeks size mass on right side of abdomen in right lumbar and iliac region extending till midline. Mass was non-tender, cystic in consistency. Complete blood count, liver and kidney function tests were normal. Magnetic resonance imaging (MRI) was done suggestive of psoas abscess of $24.7 \times 12 \times 8 \mathrm{~cm}$ extending into right thigh.

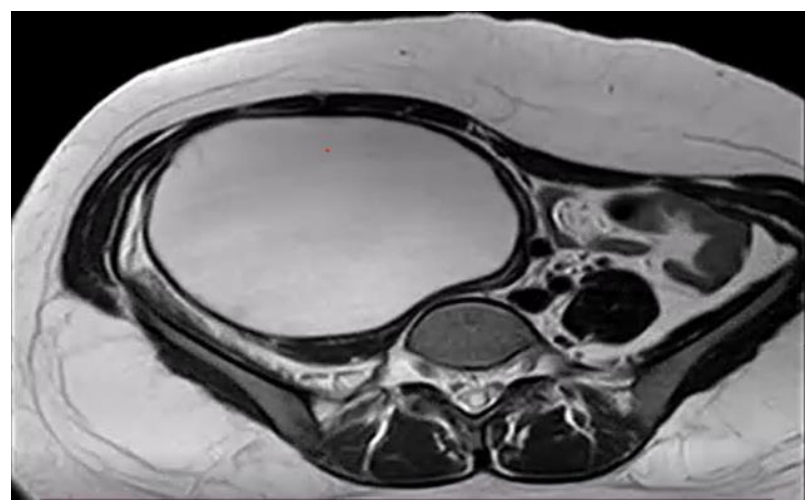

Figure 1: MRI showing right psoas abscess transverse section at the level of maximum diameter of abscess.

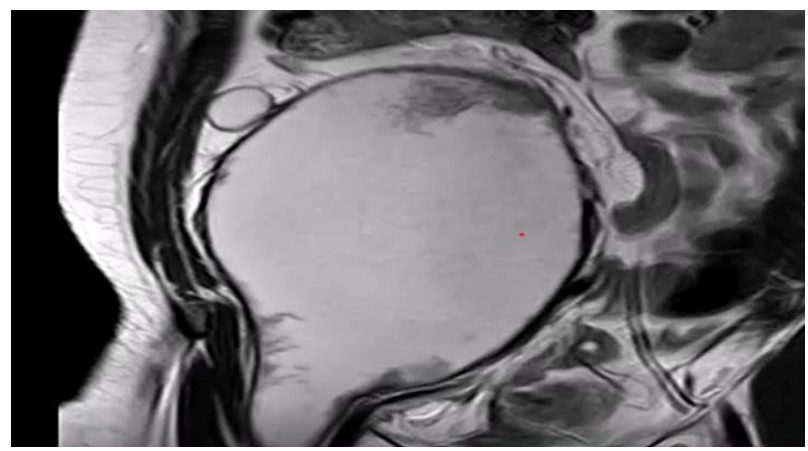

Figure 2: MRI showing psoas abscess extending into pelvis on sagittal section.

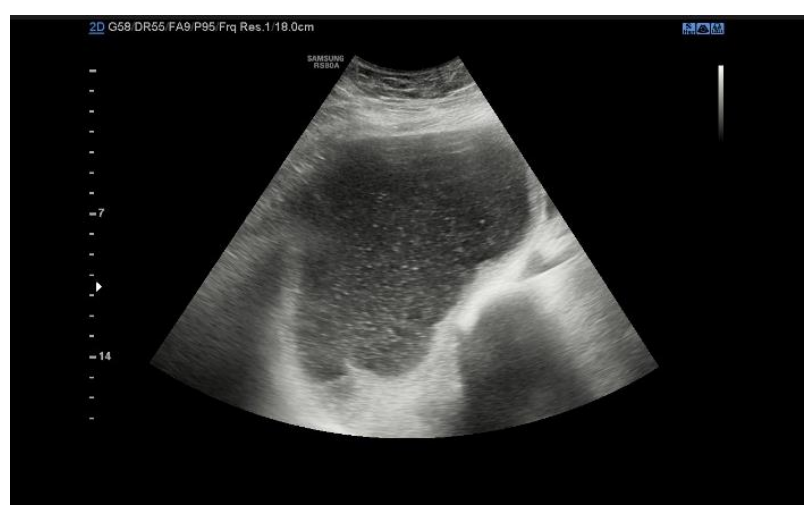

Figure 3: USG showing right psoas abscess extending till right thigh.

Ultrasound done revealed a psoas abscess and a single live fetus of 7 weeks gestation. Ultrasound guided aspiration of pus was done with pigtail catheter no. 12 Fr. and around $1800 \mathrm{ml}$ of pus drained from abscess.

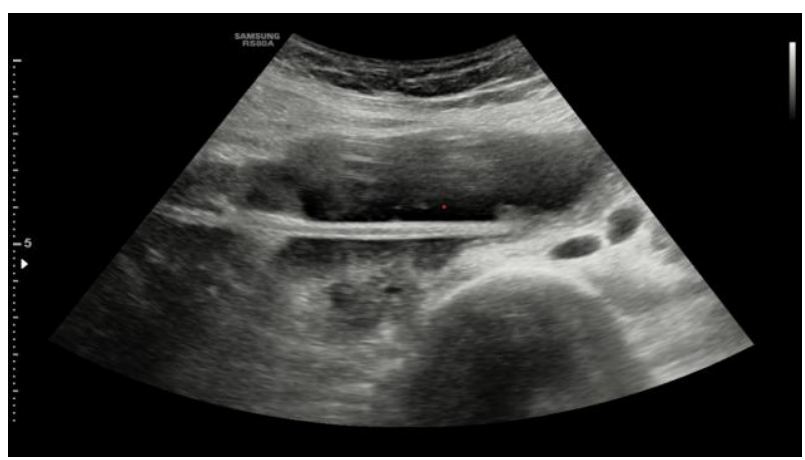

Figure 4: Pigtail catheter put in situ.

Pus sample was sent for acid fast bacilli (AFB) stain, gram stain, pus culture sensitivity and gene expert. Smear for acid fast bacilli was positive and Mycobacterium tuberculosis (MTB) with rifampicin sensitive strain was detected in gene X-pert and therefore patient was started on anti-tubercular medications. Category 1 treatment with four drugs (INH, rifampicin, pyrazinamide, and ethambutol) for 2 months followed by two drugs (INH and rifampicin) for 4 months was initiated. Pigtail catheter was kept in situ for 7 days, removed on day 8. Patient discharged on day 10 of admission.

After 2 weeks, patient was admitted again for rapidly enlarging abscess size of size $12 \times 7 \mathrm{~cm}$ on ultrasound of 11 weeks. Ultrasound guided Pigtail insertion done, around $50 \mathrm{ml}$ of pus drained. Pigtail catheter was placed in situ for 10 days. Antitubercular therapy continued. Pigtail catheter was removed and patient discharged on day 12 of admission. Patient advised regular follow up in outpatient department (OPD).

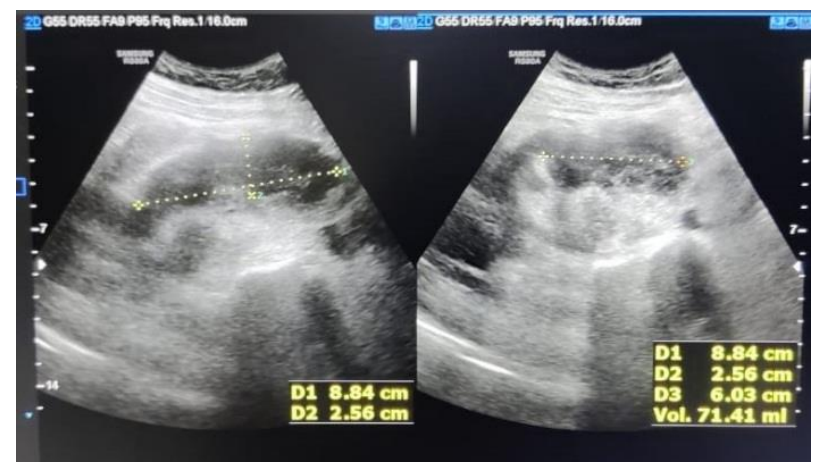

Figure 5: Psoas abscess reduced in size after drainage by pigtail catheter.

\section{DISCUSSION}

The sigmoid colon, jejunum, appendix, ureters, aorta, renal pelvis, pancreas, iliac lymph nodes, and spine are all in close proximity to the psoas muscle. Infections in these organs can thus spread to the psoas muscle. The psoas 
muscle has a dense vascular supply, which is thought to predispose it to haematogenous spread from sites of occult infection. Any infection in these regions may progress and result in a psoas abscess. These secondary psoas abscesses are generally seen in older individuals with underlying ailments. $^{4}$

Lower back pain, limping and daily fever comprise the clinical triad of psoas muscle abscess. However in our patient, there were no signs and symptoms like fever, pain in abdomen and lower back pain. Gastrointestinal and urological manifestations are seldom present. A diagnosis of psoas muscle abscess should be suspected in cases where in spite of treatment, backache and limp persist. Laboratory investigations (raised total leukocyte count and increased erythrocytic sedimentation rate (ESR)) and radiological findings aid in establishing a diagnosis. Ultrasound or computed tomography (CT) scan makes the most cost-effective diagnosis with an accuracy of 41-95\% and $95-100 \%$ respectively. ${ }^{4}$ In our patient, diagnosis was delayed due to absence of significant signs and symptoms. Abscess was diagnosed when patient went for routine first trimester dating scan.

Psoas abscess during pregnancy is a rare event. Treatment involves the drainage of the abscess and use of appropriate antibiotics. Drainage of the abscess may be done through CT or ultrasound guided percutaneous drainage or drainage at laparotomy. Percutaneous drainage is much less invasive and is effective for draining uniloculated and multiloculated abscesses with few septations. ${ }^{5}$ In our patient, abscess was drained by placing ultrasound guided pigtail catheter.

Knowledge of common pathogens should guide initial choice of antibiotics. Coverage should include staphylococcal and enteric organisms for which agents such as clindamycin, antistaphyloccocal penicillin, and an aminoglycoside may be used. Adjustments should be based on report of abscess fluid culture and sensitivity testing. ${ }^{5}$ In our patient, pus drained from abscess was positive for AFB and gene X-pert report showed mycobacterium tuberculosis and thus antitubercular therapy was started.

\section{CONCLUSION}

A rare case of psoas abscess in pregnancy presenting with nonspecific signs and symptoms managed conservatively by ultrasound guided pigtail catheter insertion and drainage of abscess multiple times and anti-tubercular therapy.

\section{Funding: No funding sources \\ Conflict of interest: None declared \\ Ethical approval: Not required}

\section{REFERENCES}

1. Kumar S, Malhotra N, Chanana C, Lal S. Psoas abscess in obstetrics. Arch Gynecol Obstet. 2009;279(2):247-9.

2. Roy S. Cold abscess in caries spine. J Indian Med Assoc. 1969;2013:240-4.

3. Mallick IH, Thoufeeq MH, Rajendran TP. Iliopsoas abscesses. Postgrad Med J. 2004;80:459-62.

4. Thomas A, Albert AS, Bhat S, Sunil KR. Primary psoas abscess - diagnostic and therapeutic considerations. Br J Urol. 1996;78(3):358-60.

5. Kumar S, Malhotra N, Chanana C, Lal S. Psoas abscess in obstetrics. Arch Gynecol Obstet. 2009;279(2):247-9.

Cite this article as: Chavan NN, Ammara U, Dias Z, Boob M. A rare case report of psoas abscess in pregnancy in a tertiary centre. Int $\mathrm{J}$ Reprod Contracept Obstet Gynecol 2022;11:272-4. 